\title{
Análise da correlação do Índice de Helkimo com a função respiratória no pré e pós-operatório de pacientes submetidos à cirurgia cardíaca. Estudo Piloto Correlation analysis of Helkimo index with lung function in pre-and postoperative patients undergoing cardiac surgery. Pilot Study
}

\author{
Cristiane Delgado Alves Rodrigues ${ }^{1}$, Caroline Andréia Pizano ${ }^{2}$, Melina Tarossi ${ }^{2}$ Ligia dos Santos Roceto ${ }^{1}$, Desanka
}

Dragosavac ${ }^{3}$

1 Fisioterapeuta do $\mathrm{HC}$ UNICAMP, Supervisora do Curso de Especialização em Fisioterapia Respiratória em UTI de Adultos.

2 Fisioterapeuta, Especialista em Fisioterapia Respiratória em UTI de Adultos do HC UNICAMP.

3 Médica Intensivista do $\mathrm{HC}$ UNICAMP, Coordenadora do Curso de Especialização em Fisioterapia Respiratória em UTI de Adultos.

Hospital das Clínicas da Universidade Estadual de Campinas- UNICAMP, Campinas- SP.

Número de aprovação do CEP da Instituição: protocolo 435/2009.

\section{ENDEREÇO PARA}

CORRESPONDÊNCIA:

Cristiane Delgado Alves Rodrigues

Av. Jesuíno Marcondes Machado, 2257 - CEP: 13.090723 - Campinas/ SP. crist_rodrigues@yahoo.com.br / (19)32533454

APRESENTACÃ̃O: abr. 2010

ACEITO PARA PUBLICAÇ̃̃O: set. 2010
Resumo: Avaliar a articulação temporomandibular (ATM) e a função respiratória no pré e pós-operatório de pacientes submetidos à cirurgia cardíaca eletiva, com intubação orotraqueal (IOT) e verificar se há correlação entre o Índice de disfunção clínica craniomandibular (IDCCM) com a função respiratória. Foram avaliados pacientes no pré, primeiro e segundo dias de pós-operatório (PO1, PO2) de cirurgia cardíaca com até 24 horas IOT. Na avaliação da ATM foi utilizado o IDCCM e para a avaliação respiratória foram utilizados a cirtometria axilar, xifoideana e umbilical, ventilometria, incluindo capacidade vital (CV), volume minuto $(\mathrm{VM})$ e frequência respiratória (FR). Completaram o estudo 13 pacientes. Não foi encontrada diferença estatística entre o IDCCM nos diferentes períodos de avaliação. Houve diminuição significativa dos valores de cirtometria nas três medidas e da CV com $p<0,001$. A FR apresentou aumento significativo, com $p=0,01$. Foi encontrada correlação negativa entre o IDCCM no PO1 e a cirtometria axilar no PO1; correlação negativa entre o IDCCM no PO2 e a cirtometria xifoideana no PO2, e correlação positiva entre o IDCCM no PO2 e FR no PO2. Pode-se concluir que o IDCCM não se alterou significativamente após intubação orotraqueal ao longo dos tempos estudados. A função respiratória apresentou diminuição dos volumes e capacidades pulmonares. A cirtometria axilar e xifoideana, assim como a FR apresentaram correlação com o IDCCM em diferentes tempos.

DescRITORES: transtornos da articulação temporomandibular, cirurgia torácica, sistema estomatognático, intubação, medidas de volume pulmonar.

AвSTRAст: To evaluate the temporomandibular joint (TMJ) and respiratory function in pre-and postoperative patients undergoing elective cardiac surgery with tracheal intubation $(\mathrm{TI})$ and verifying the correlation between Clinical dysfunction index (CDI) with lung function. Patients were evaluated during the first and second days after surgery (PO1, PO2) of cardiac surgery with 24 hours of intubation. For the evaluation of the TMJ was used CDI and for respiratory function were used cirtometry axillary, xiphoid and umbilical, and respirometry, including vital capacity (VC), minute volume (MV) and respiratory rate (RR). Thirteen patients completed the study. There was no statistical difference between the CDI at different stages of evaluation, and when evaluated the 5 sub-items, only the item assessing function showed significant result, $p=0.01$. There was a significant decrease of the values of circumferences in the three measures and CV $p<0.001$. Negative correlation was found between the $\mathrm{CDI}$ and circumference in PO1 axillary; negative correlation between the CDI in $\mathrm{PO} 2$ and $\mathrm{PO} 2$ in xifoideana circumference, and a positive correlation between the $\mathrm{CDI}$ in $\mathrm{PO} 2$ and PO2 in the FR. It can be concluded that the CDI did not change significantly after tracheal intubation through the ages studied. Respiratory function showed a decrease in volumes and lung capacities. Cirtometry axillary and xifoideana and the FR were correlated with the CDI at different times.

KEY WORDs: temporomandibular joint disorders, thoracic surgery, stomatognathic system, intubation, lung volume measurements. 


\section{INTRODUÇÃO}

O termo desordem temporomandibular (DTM) é utilizado para designar alterações patológicas e funcionais que afetam a articulação, os músculos mastigatórios e outras partes do sistema estomatognático ${ }^{1}$. Constitui-se de desordens caracterizadas por dor pré-auricular, na articulação ou músculos, com limitações na amplitude de movimento, ruídos articulares e desvios durante a abertura e fechamento bucal ${ }^{2}$

A etiologia da DTM é multifatorial ${ }^{3,4}$, incluindo traumas diretos na face, sobrecarga na articulação e/ou músculos mastigatórios durante tempo prolongado, hábitos parafuncionais, alterações oclusais, doenças reumáticas, fatores emocionais, alterações posturais, e mais recentemente disfunções respiratórias ${ }^{5,6}$.

Bérzin e Corrêa ${ }^{7}$ em revisão bibliográfica estudaram a relação da DTM com as disfunções respiratórias, ressaltando a interferência do uso dos músculos acessórios, padrão de respiração costal e respiração bucal e verificaram que tais condições estão intimamente ligadas a alteração da dinâmica muscular e consequentemente com as DTM's.

Por sua vez, as cirurgias torácicas interferem diretamente na mecânica pulmonar, sendo que as alterações pulmonares no pós-operatório trazem como consequência um padrão respiratório restritivo. Observa-se assim redução do volume corrente, da capacidade vital, da capacidade residual funcional e da pressão parcial de oxigênio ${ }^{8}$.

Os pacientes que realizam cirurgia cardíaca são submetidos ao procedimento de laringoscopia. Segundo Aiello ${ }^{9}$, a manobra de laringoscopia faz com que a ATM perca sua proteção devido à perda do tônus dos músculos adjacentes, podendo desenvolver temporariamente DTM nesses pacientes.

Considerando os fatores etiológicos para as DTM's pode-se associar que a laringoscopia ocasiona abertura excessiva da boca através de aplicação de forças diretas, podendo causar estiramento da cápsula e ligamentos ou ainda luxação da mandíbula ${ }^{10}$. Nem sempre os pacientes submetidos à IOT apresentam extubações imediatas, o que implica em complicações como fibroses e aderências da ATM $^{11}$
Segundo Martin et al. ${ }^{12}$ o acompanhamento a médio e longo prazo de pacientes submetidos a IOT revelou que a dor no sétimo pós-operatório está inversamente correlacionada à menor distância intercisal, porém não esclarece se a IOT é capaz de provocar a DTM a médio e longo prazo.

Diante da escassez de estudos relacionando DTM's e IOT, o objetivo principal deste estudo foi avaliar a ATM e a função respiratória no pré e pós- operatório de pacientes submetidos à cirurgia cardíaca eletiva. E como objetivo secundário, verificar se há correlação entre o IDCCM com a função respiratória.

\section{METODOLOGIA}

Trata-se de um estudo prospectivo e não intervencionista dos pacientes no pré e pós-operatório de cirurgia cardíaca eletiva, submetidos à anestesia geral e IOT.

Os pacientes foram selecionados de forma contínua através de amostra de conveniência. Foram incluídos pacientes que permanecessem intubados por até 24 horas, com idade entre 18 e 80 anos, internados para cirurgia cardíaca eletiva com IOT. Os critérios de exclusão foram: pacientes que necessitassem de tempo de intubação maior que 24 horas no pós-operatório; que não tivessem dentição nem prótese dentária; que apresentassem déficit cognitivo, ou antecedente neurológico que impossibilitasse a avaliação.

O estudo foi realizado no período de maio a novembro de 2009. Para a seleção, os pacientes foram entrevistados expondo o objetivo e resultados esperados da pesquisa e, caso o paciente concordasse em participar, era solicitada a assinatura do Termo de Consentimento Livre e Esclarecido. O projeto foi devidamente aprovado pelo CEP da Instituição sob protocolo 435/2009.

Os pacientes foram avaliados no pré-operatório na Enfermaria de Cardiologia, e reavaliados no primeiro e segundo dias de pós-operatório na UTI do HC da UNICAMP.

A avaliação da ATM foi realizada através do Índice de Disfunção Clínica Craniomandibular - IDCCM (ou índice de Helkimo) ${ }^{4}$, que é dividido em 5 subitens. O primeiro avalia a mobilidade mandibular, no qual um maior escore representa piora da mobilidade. Os ou- tros subitens avaliam dor ao movimento, dor na articulação, alteração na função e dor muscular. É pontuado de 0 a 25 classificando a severidade em: nenhuma disfunção (0 pontos), disfunção suave (1 a 4 pontos), moderada (5 a 9 pontos) ou severa (10 a 25 pontos). Esse índice não fornece classificação diagnóstica, apenas avalia a severidade dos sinais de DTM ${ }^{13}$. Entretanto os indivíduos selecionados para essa pesquisa não foram previamente diagnosticados como portadores de DTM por outras ferramentas, índices ou escores.

Para avaliação do IDCCM o paciente encontrava-se em posição de Fowler. A medida da abertura bucal foi realizada com paquímetro $\left(\mathrm{ICE}^{\circledR}\right)$ posicionado entre os incisivos superiores e inferiores, pedindo para que o participante abrisse a boca ao máximo. Já a protrusão mandibular foi obtida através da distância entre os incisivos com a mandíbula protruída. E a medida de lateralidade mandibular para a direita e para a esquerda, realizada com o paquímetro posicionado através das linhas médias dos incisivos superiores e inferiores. Para avaliação dos ruídos foi utilizado um estetoscópio $\left(\mathrm{BD}^{\circledR}\right)$, posicionado sobre a ATM durante a abertura e fechamento bucal ${ }^{14}$.

A avaliação da função respiratória incluiu a cirtometria axilar, xifoideana e umbilical, aferidas com fita métrica graduada em centímetros nas quais se mensuraram a diferença entre a inspiração e a expiração máximas, realizadas com o paciente na posição de Fowler. Além disso, avaliou-se a ventilometria, através do ventilômetro Ferraris Wright $M K{ }^{\circledR}{ }^{\circledR}$, no qual foram mensuradas a capacidade vital (CV), o volume minuto (VM) e a frequência respiratória (FR). A CV foi medida a partir de uma inspiração até a capacidade pulmonar total seguida de expiração até o volume residual. As medidas foram realizadas por no máximo seis vezes, até que fossem obtidos três valores com variação menor que $5 \%$, sendo utilizado o maior valor obtido ${ }^{15}$. Para aferição do VM, o paciente foi orientado a respirar no ritmo normal com auxílio de um bocal e quando o paciente estivesse adaptado, era iniciada a contagem da FR em um minuto. Ambas as manobras realizadas com clip nasal e com o paciente na posição de Fowler. Vale ressaltar que todos os pacientes faziam uso de drenos de pericárdio e mediastino até o PO2. Dessa forma, as medidas eram feitas após a anal- 
gesia prescrita, para que a dor não fosse fator limitante na resposta do paciente.

\section{ANÁLISE ESTATÍS-} TICA

Os dados foram submetidos à análise descritiva. Foi encontrada ausência de distribuição normal das variáveis pelo teste de Kolmogorov-Smirnov. Para avaliar as variáveis contínuas foram utilizados os testes de Wilcoxon e de Friedman. A correlação entre as variáveis foi realizada pelo teste de Spearman. O nível de significância utilizado foi de $p<0,05$. Os dados foram processados pelo programa SPSS versão 15.0.

\section{Resultados}

Foram avaliados no total 34 pacientes, dos quais 21 foram excluídos. Sendo assim, completaram o estudo 13 pacientes cujas características se encontram na tabela 1.

Com relação ao escore total do IDCCM não foi encontrado diferença estatisticamente significativa entre seus valores nos diferentes períodos de avaliação, com $p=0.09$.

Com relação à avaliação respiratória, a cirtometria nas três medidas de referência apresentou resultados estatisticamente significativos, com $p<0,001$ para todas as medidas, evidenciando diminuição em seu valor, quando comparada nos três períodos. A CV e o VM apresentaram diminuição nos seus valores, sendo estatisticamente significativo apenas para $C V$, com $p<0,001$. Os resultados referentes à FR mostraram aumento de seu valor, estatisticamente significativo, com $p=0,01$. Durante a avaliação era questionada a dor e se o pacientes a relatasse aguardava-se o uso da analgesia para prosseguir a avaliação. Esses dados encontram-se na tabela 2.

Dentre as correlações entre o IDCCM e a função respiratória, houve correlação negativa moderada entre o IDCCM no PO1 e a cirtometria axilar no PO1, correlação negativa forte entre o IDCCM no PO2 e a cirtometria xifoideana no PO2. E encontrou-se correlação positiva moderada entre o IDCCM no PO2 e FR no PO2. Os coeficientes de correlação se encontram na tabela 3
Tabela 1 Características da população estudada.

\begin{tabular}{ll}
\hline Variáveis & $\mathrm{n}=13$ \\
\hline Idade $\left(\right.$ mediana $-1^{\circ} \mathrm{Q}$ e $\left.3^{\circ} \mathrm{Q}\right)$ & $58(51,5 / 66,0)$ \\
Sexo $(\mathrm{F} / \mathrm{M})$ & $3(21,4 \%) / 10(71,4 \%)$ \\
Cirurgia (RVM/TV) & $8(57,1 \%) / 5(35,7 \%)$ \\
Extubação (POi/PO1) & $12(85,7 \%) / 1(7,1 \%)$ \\
Tipo de prótese (S/C/D) & $2(14,3 \%) / 5(35,7 \%) / 6(42,9 \%)$
\end{tabular}

$1^{\circ} \mathrm{Q}=$ primeiro quartil; $3^{\circ} \mathrm{Q}=$ terceiro quartil; $\mathrm{F}=$ feminino; $\mathrm{M}=$ masculino;

RVM=revascularização miocárdio; TV=troca de válvula; $\mathrm{POi}=$ pós operatório imediato; PO1=primeiro dia pós operatório; $\mathrm{S}=$ prótese superior; $\mathrm{C}=$ prótese completa (superior e inferior); $\mathrm{D}=$ dentição própria.

Tabela 2 Comportamento das variáveis analisadas nos três períodos, representados pela mediana, $1^{\circ} \mathrm{Q}$ e $3^{\circ} \mathrm{Q}, \mathrm{n}=13$.

\begin{tabular}{lllll}
\hline Variáveis & Pré & PO1 & PO2 & P valor \\
\hline Escore Total IDCCM & $2(1 ; 2)$ & $1(1 ; 2)$ & $1(1 ; 2)$ & 0,09 \\
IDCCM & & & & \\
ADM & $5(1 ; 5)$ & $1(1 ; 5)$ & $1(1 ; 5)$ & 0,47 \\
Dor ao movimento & $0(0 ; 0)$ & $0(0 ; 0)$ & $0(0 ; 0)$ & 0,09 \\
Dor na Articulação & $0(0 ; 0)$ & $0(0 ; 0)$ & $0(0 ; 0)$ & 0,36 \\
Função & $0(0 ; 1)$ & $0(0 ; 0)$ & $0(0 ; 0)$ & $0,01^{*}$ \\
Dor Muscular & $0(0 ; 1)$ & $0(0 ; 0)$ & $0(0 ; 0)$ & 0,10 \\
\hline Avaliação Respiratória & & & & \\
\hline Cirt Axilar (cm) & $3,5(2 ; 4)$ & $1,5(1 ; 2)$ & $2,5(2 ; 3,5)$ & $0,00^{*}$ \\
Cirt Xifoideana (cm) & $3(2 ; 3,2)$ & $1(1 ; 5)$ & $2(1 ; 2,5)$ & $0,00^{*}$ \\
Cirt Abdominal (cm) & $3,5(2,7 ; 4,2)$ & $2,5(1,5 ; 3)$ & $3(2 ; 3,5)$ & $0,00^{*}$ \\
CV (L) & $2,8(2,1 ; 3,6)$ & $1,1(9,0 ; 1,8)$ & $1,2(9,2 ; 2,4)$ & $0,00^{*}$
\end{tabular}

$\mathrm{VM}(\mathrm{L})$

$13,4(11,4 ; 15,8) 13,0(11,1 ; 16,1) 11,4(9,6 ; 15,5) \quad 0,87$

FR

$14(9 ; 16)$

$17(11 ; 22)$

$19(12 ; 26)$

$0,01 *$

$1^{\circ} \mathrm{Q}=$ primeiro quartil; $3^{\circ} \mathrm{Q}=$ terceiro quartil; $\mathrm{ADM}=$ amplitude de movimento; $\mathrm{CV}=$ capacidade vital; $\mathrm{VM}=$ volume minuto; $\mathrm{FR}=$ freqüência respiratória; Pré= pré-operatório; $\mathrm{POi}=$ ós operatório imediato; PO1=primeiro dia pós operatório; $\mathrm{PO} 2=$ segundo dia de pós-operatório; IDCCM: Índice de disfunção clínica craniomandibular.

Tabela 3 Correlação entre o IDCCM com a cirtometria toracoabdominal e com a FR.

\begin{tabular}{lcc}
\hline Variáveis correlacionadas & $R$ & p valor \\
\hline IDCCM PO1 X Cirtometria Axilar PO1 & $-0,619^{*}$ & 0,042 \\
IDCCM PO2 X Cirtometria Xifoideana PO2 & $-0,697^{* *}$ & 0,008 \\
IDCCM PO2 X FR PO2 & $0,668^{*}$ & 0,025
\end{tabular}

** Forte correlação; * moderada correlação; FR= frequência respiratória; $\mathrm{PO} 1=$ primeiro pósoperatório; $\mathrm{PO} 2=$ segundo pós-operatório. 


\section{DISCUSSÃO E CONCLUSÃO}

De forma geral, vários fatores têm sido relacionados à etiologia das DTM's, sendo que alguns autores têm associado a IOT com alterações na ATM. Nesse sentido, Lipp et al. ${ }^{16}$ examinaram a influência da IOT com anestesia geral nas lesões da ATM em 100 pacientes submetidos a diversas cirurgias (exceto de cabeça e pescoço) sendo 50 sob anestesia local e 50 sob anestesia geral. Os autores concluíram que $66 \%$ dos pacientes submetidos à IOT e anestesia geral obtiveram redução de $35 \%$ da capacidade de abertura bucal no primeiro dia de pós-operatório, além disso, dois dos 50 pacientes apresentaram dor, $80 \%$ dos intubados apresentaram estalidos (valor 20\% acima do pré-operatório); dois dos 50 intubados desenvolveram alterações oclusais e outros dois tiveram redução na distância mínima entre as bordas oclusais dos incisivos superiores e inferiores.

Nesse mesmo contexto, Agro et al. ${ }^{10}$, avaliaram a abertura máxima da boca o desvio da mandíbula durante abertura ou fechamento, a presença de estalido e dor à palpação em pacientes submetidos às cirurgias, com IOT, no pré-operatório e no primeiro dia de pós-operatório. Os pacientes com pelo menos duas das alterações acima eram considerados portadores de DTM. Como resultado, observou-se que $44 \%$ dos pacientes com diagnóstico de DTM tiveram piora, e que $5 \%$ dos que não tinham sintomas passaram a apresentar.

No presente estudo quando analisado o escore total do IDCCM pôde-se observar que os pacientes que se mantiveram intubados por até 24 horas não apresentaram alterações significativas da ATM, mesmo os pacientes que já apresentavam algum grau de DTM no pré-operatório, resultados que diferem dos estudos encontrados na literatura.

Outros fatores que podem justificar os nossos achados é a composição da amostra, que em sua maioria foi do gênero masculino, e faixa etária entre 39 a 78 anos, variáveis que se diferem do que a literatura traz como gênero e faixa etária prevalentes para DTM, como mostra o estudo de Lopes ${ }^{17}$. Neste estudo avaliou-se a prevalência de sinais e sintomas de DTM em 206 indivíduos os quais foram divididos por idade em quatro grupos diferentes. Observou-se que $39 \%$ da população apresentavam sinais e sintomas de DTM, sendo $67 \%$ para o gênero feminino e $33 \%$ para o masculino, ou seja, com maior prevalência em mulheres e a faixa etária de 26 a 45 anos.

Outro estudo com resultados que diferem do presente trabalho foi o de Martin et al. ${ }^{12}$ que avaliaram os fatores de risco para DTM após intubação em 122 pacientes, no pré-operatório, no sétimo e décimo quarto dias de pós-operatório. Os autores concluíram que a menor amplitude de movimento da ATM, sexo feminino e idade são fatores de risco para DTM transitória em pacientes submetidos à IOT

Taylor et al. ${ }^{18}$ encontraram resultados semelhantes ao presente estudo no que se refere à incidência de DTM pós intubação, já que observaram que a incidência de DTM foi menor que $5 \%$. Os autores avaliaram 62 pacientes de dez a 69 anos no pré-operatório, no primeiro e segundo dias de pós-operatório, através de um questionário e exame clínico. Destes, 24 pacientes já tinham DTM previamente, e apenas três pacientes apresentaram alteração dos sintomas após IOT, o restante não mostrou aumento dos sinais e sintomas.

Com relação à avaliação respiratória houve diminuição estatisticamente significativa da cirtometria nas três medidas avaliadas e da $C V$, além do aumento na FR. Estes fatores são esperados após cirurgia cardíaca, pois a dor, consequência inevitável deste procedimento limita a expansibilidade e a mobilidade torácica, inibe a inspiração profunda e associado à esternotomia mediana contribui para a deterioração do quadro. Neste contexto, a presença de drenos implica diretamente na manutenção dos baixos volumes pulmonares. Além disso, o quadro de disfunção pulmonar pós cirurgia cardíaca é secundário à utilização de circulação extracorpórea (CEC), indução anestésica e trauma cirúrgico ${ }^{19,20}$.

A literatura nos mostra que a DTM pode estar relacionada às disfunções respiratórias, e que essa relação se dá principalmente pelo uso excessivo da musculatura inspiratória acessória que associada ao padrão respiratório apical pode levar às alterações posturais, que por sua vez afetam diretamente a posição da ATM $^{9}$.

Nesse contexto, Pasinato et al. ${ }^{6}$, avaliaram a presença de patologia respiratória prévia, tipo de padrão ventilatório, modo respiratório e mobilidade torácica em 35 indivíduos divididos em dois grupos: pacientes com DTM e assintomáticos. Como resultado observou-se maior incidência de doenças obstrutivas nos pacientes com DTM. O modo respiratório predominante foi o nasal em ambos os grupos, e o modo bucal não foi observado nos assintomáticos, apresentando-se em $30 \%$ do grupo DTM. Quanto ao padrão respiratório, no grupo DTM foi predominante o apical, e nos assintomáticos o diafragmático.

No presente estudo as correlações entre o IDCCM e as cirtometrias axilar e xifoideana evidenciaram que a piora dessas (diminuição da mobilidade torácica) está relacionada ao aumento do IDCCM, que significa piora dos sintomas de DTM. Já quando se correlaciona o IDCCM com a FR observou-se que o aumento da FR está relacionado também com a piora dos sintomas de DTM no PO2.

Fatores como dor, presença de drenos e esternotomia mediana, contribuem para as limitações da expansibilidade torácica, ocasionando na maioria dos pacientes um padrão respiratório superficial e apical, que pode ser mensurado pela cirtometria, e justificar as correlações encontradas entre às alterações da ATM e função respiratória ${ }^{20}$. É esperado que as alterações na função pulmonar em cirurgia cardíaca se normalizem a partir do PO5 ${ }^{21}$, assim como as disfunções de ATM. Diante disso, se os pacientes deste estudo tivessem sido acompanhados por mais tempo no pós-operatório tais correlações de ATM e expansibilidade torácica poderiam não ter sido encontradas. Além disso, outra limitação do estudo que pode ter influenciado nos resultados foi a não mensuração da dor relatada pelo paciente, sendo esta apenas avaliada pelo IDCCM, ressaltando-se que a analgesia padrão foi estabelecida durante todo o período de coleta.

Conclui-se que utilizando o IDCCM não houve alteração significativa dos 
sintomas de DTM após IOT na população estudada. Com relação à função respiratória houve diminuição dos vo- lumes e capacidades pulmonares, fato consolidado pela literatura nos pacientes em pós-operatório de cirurgia cardíaca.
E foi encontrada tendência à piora do IDCCM correlacionado à piora da função respiratória no pós-operatório.

\section{REFERÊNCIAS}

1 Bigaton DR, Almeida AFN, Berni KCS, Pedroni CR, Gonçalves RN, Bérzin F. Utilização de diferentes estimulações elétricas para o tratamento da dor em mulheres com disfunção temporomandibular. Rev Bras Fisioter. 2008;12(6):476-81.

2 Medlicott MS, Harris SR. A systematic review of the efectiveness of exercise, manual therapy, electrotherapy, relaxation training, and biofeedback in the management of temporomandibular disorder. Physical Therapy. 2006;86(7):955-73.

3 Rodrigues ETT, Avaliação da articulação temporomandibular pós intubação orotraqueal. [Dissertação]. Campinas: São Leopoldo Mandic, 2008.

4 Chaves TC, Oliveira AS de, Grossi DB. Principais instrumentos para avaliação da disfunção temporomandibular, parte I: Índices e questionários; uma contribuição para a prática clínica e de pesquisa. Rev Fisioter Pesqui. 2008;15(1):121-31.

5 Diagnosis and managment of temporomandibular disorders and relate muscoloskeletal disorders. Guidelines. 2006:1-12.

6 Pasinato F, Corrêa ECR, Peroni ABF, Avaliação da Mecânica ventilatória em indivíduos com disfunção têmporo-mandibulares e assintomáticos. Rev Bras fisioter. 2006;10(3):285-89.

7 Bérzin F, Corrêa ECR. Temporomandibular Disorder in dysfunctional breathing. Braz J Oral Sci. 2004;3(10):498-502.

8 Rodrigues, CDA. Efeitos do suporte ventilatório com pressão controlada e volume controlado na função pulmonar dos pacientes submetidos à cirurgia cardíaca com circulação extracorpórea. Campinas: Universidade Estadual de Campinas, 2009.

9 Aiello G, Metclaf L. Anaesthetic implications of Temporomandibular joint disease. Can J Anaesth. 1992;39(6):610-16.

10 Agro F, Salvinelli F, Casale M, Antonelli S. Temporomandibular joint assessment in anaesthetic practice. BR J Anaesth. 2003 90(5):707-08.
11 Rattan V. Prolonged Temporomandibular Joint Dislocation in an Unconscious Patient After Airway Manipulation. Anesthesia and analgesia. 2006;102:1287-99.

12 Martin MD, Wilson KJ, Ross BK, Souter K. Intubation Risk Factors for Temporomandibular Joint/Facial Pain. Anesthesia Progress. 2007;54(3):109-14.

13 Almeida et at. Índice de Helkimo e Craniomandibular para diagnóstico de desordens têmporo-mandibulares Revisão de Literatura. Rev. Cir. Traumatol. Buco-Maxilo-Fac. 2005;5(3):9-16.

14 Gonzalez BDA. Abordagem Interdisciplinar das Disfunções Temporomandibulares. Ed. Manole: São Paulo, 2005. 246 p.

15 Junior JFF, Paisani DM, Franceschini j, Chiavegato LD, Faresin SM. Pressões respitarórias máximas e capacidade vital: comparação entre avaliações através de bocal e de máscara facial. J Bras Pneumol, 2004;30(6):515-20.

16 Lipp M, von Domarus H, Daubländer M, Leyser KH, Dick W. Effects of intubation anesthesia on the temporomandibular joint. Anaesthesist. 1987;36(8):442-45.

17 Lopes, MR. Prevalência dos sinais e sintomas de disfunção temporomandibular na população de Teresina-PI no ano de 2005 [dissertação]. Campinas: Centro de Pós-Graduação São Leopoldo Mandic; 2007.

18 Taylor RC, Walter LW, Hendrixson RA. Temporomandibular joint problems in relation to the administration of general anesthesia. J Oral Surg. 1968;26(5):327-29.

19 Luchesa CA, Greca FH, Guarita-Souza LC,Santos JLV, Aquim EE. Papel da eletroanalgesia na função respiratória de pacientes submetidos à operação de revascularização do miocárdio. Rev Bras Cir Cardiovasc. 2009;24(3):391-96.

20 Renault JÁ, Costa-Val, R, Rosseti MB. Fisioterapia respiratória na disfunção pulmonar pós-cirurgia cardíaca. Rev Bras Cir Cardiovasc. 2008;23(4):562-569.

21 Giacomazzi CM, Lagni VB, Monteiro MB. A dor pós operatória como contribuinte do prejuízo na função pulmonar em pacientes submetidos à cirurgia cardíaca. Braz J Cardiovasc Surg. 2006;21(4):386-92. 